\title{
The Solution to the Dark Energy Mystery in the Universe of Four Distance Dimensions
}

\author{
Ahti Rahikainen \\ Merkkipuuntie 2 L 39, Vantaa, Finland \\ Email: ahrahik.zz@kolumbus.fi
}

How to cite this paper: Rahikainen, A. (2021) The Solution to the Dark Energy Mystery in the Universe of Four Distance Dimensions. World Journal of Mechanics, 11, 95-110.

https://doi.org/10.4236/wjm.2021.115008

Received: March 1, 2021

Accepted: May 25, 2021

Published: May 28, 2021

Copyright $\odot 2021$ by author(s) and Scientific Research Publishing Inc. This work is licensed under the Creative Commons Attribution International License (CC BY 4.0).

http://creativecommons.org/licenses/by/4.0/

\begin{abstract}
This study is a continuation of the solution to the dark matter mystery in the earlier study. In that study, the theoretical four-dimensional mass generates similar galaxy rotations as were measured. This proves that the theory of four-dimensional mass is correct, especially as all the calculations are basic mechanics and easy to understand. Most of the mass and energy in the Universe is unknown. The common knowledge is that the ordinary mass is only about $5 \%$, the dark matter that rotates the galaxies comprises $26 \%$, and dark energy that expands the Universe at an accelerating speed is $69 \%$. This study solves the mystery of dark energy by using the structure of the four-dimensional Universe. The theory of the Universe expanding at an accelerating speed has been based on the redshift measurements, in which the redshift of light from distant galaxies increases at an accelerating rate. Therefore, it has been inferred that the whole Universe expands at an accelerating speed. In this study, the model of the Universe is the surface volume of the four-dimensional spherical Universe. This type of structure of the Universe creates the same kind of an accelerating redshift increase which has been measured. In order to prove this theory, the model of the surface volume of the four-dimensional Universe was constructed, the equation of the redshift caused by this Universe was solved, and the theoretical equation was shown to be the same as the measured redshift in the Universe. The measured redshift in the Universe was obtained from the derivative of the model of the expanding Universe. A similar model of the Universe has been published by NASA. The four-dimensional model of the Universe in this study yielded the Universe that was found to have decelerating expansion at this point of time, and a Big Bang that was not very big.
\end{abstract}

\section{Keywords}

Dark Matter, Dark Energy, Expansion of Universe, Big Bang,

Four-Dimensional Sphere, Four-Dimensional Matter, Atom Theory 


\section{Introduction}

The Sun and other stars that are visible in the sky are a part of the Milky Way galaxy. The Milky Way is a typical spiral galaxy that consists of a thin rotating disk of stars, and in its middle, there is a bulge of densely located stars. The stars of the Milky Way rotate around the center of the galaxy at a certain rotational speed. The Sun and the Earth are located in the middle of the disk of the Milky Way at a distance of 25,000 light-years from its center, and they rotate around the center at a speed of approximately $220 \mathrm{~km} / \mathrm{s}$. Edwin Hubble was the first astronomer who inferred that spiral nebulae are galaxies located at great distances from the Milky Way, publication 1929. In publication [1] 1978, it was found that the masses of galaxies were underestimated by a factor of 10 or more, and therefore, the density of the universe was also underestimated by the same factor. The speeds of stars in these galaxies have been measured based on the redshifts of the light they emit. The speed of a star results in a change of the wavelength, which is called redshift. In the Universe all stars at great distances have the change of wavelength towards red, and therefore, it has been inferred that the Universe is expanding. By using the redshifts, Vera Rubin measured the rotating speeds of galaxies and found that the stars rotate in the outer part of galaxy approximately at the same speed, publication [2] 1983. The speed distribution is zero at the center of galaxy, after which it rapidly increases until at a certain value it remains constant. The matter of a spiral galaxy is concentrated into a great, dense bulge at the center of the galaxy, and the rest of the galactic matter forms a large rotating spiral disk. The gravitational force of this kind of galactic matter distribution reduces approximately inversely proportional to the square of the distance from the center of the galaxy. Therefore, the visible and dark matter in the center bulge and disk of the galaxy does not only result in the constant value speed distribution. There must be a great amount of some other type of dark matter affecting the rotation of stars. The question is what type of mass distribution induces the constant rotational speed distribution on the outer part of galactic disk. It should probably be a ball-shaped dark matter formation. It is inferred that the missing dark matter could even comprise $90 \%$ of the total mass of the galaxy, and it would probably be planet-like heavenly bodies, gas and dust clouds concentrated into a huge ball or halo around the disk of stars. This type of galactic structure may possibly induce the redshift distribution. It was expected to be so, and accordingly these types of heavenly bodies around the galactic disk were searched for. The highly accurate images of the space telescope Hubble made it possible to observe dim objects at great distances. However, the results of these observations did not meet the expectations. A sufficient amount of dark matter above the galactic disk was not found. The measurements of the space telescope Hubble have not given support to this theory, publication [3] 1995. It seems that dark matter in the halo of the galaxy is not so massive that it could explain the constant speed distribution. The search for the dark matter halo continues in publications [4] 2010 and [5] 2012. After that, the search for dark matter has 
primarily concentrated no fundamental particles. It must be a fundamental particle that has mass and force of gravity. The particle must not have any other forces because otherwise it would have been observed by now. Fundamental particles like this have been searched in mine shafts that enable minimal disturbing factors. Richard Panek has written on the latest difficulties in the research of the Universe, publication [6] 2020. Astronomers have calculated how fast the universe is expanding. Two different calculation methods have two different results. The cause of the discrepancy may be the fact that physics has new unknown phenomena. The mystery of dark matter in the rotation of galaxies is a common subject matter in the cosmology textbooks, publication [7]. The publication [8] presented a new idea that the dark matter is located at the fourth distance dimension above the center of the galaxy. In the same manner as a structure of three dimensions can be drawn in the cross-sections of two dimensions, a structure of four dimensions can also be drawn in the cross-sections of two or three dimensions. Therefore, the determination of the location of dark matter in the fourth dimension is no problem. The study in the publication [8] contains the solution to the dark matter mystery of spiral galaxies by using the space of four distance dimensions $x, y, z, x^{\prime}$, in which $x^{\prime}$ is the fourth distance dimension. The calculation of the galaxy rotation has been presented in the space of four dimensions by using the two and three dimensional sections $x, x^{\prime}$ and $x, y, x^{\prime}$. The four-dimensional mass $M$, which generates the main gravitation field of the galaxy, is located in the fourth dimension at the distance $x^{\prime}=X^{*}$ and the other dimensions are zero $x=0, y=0, z=0$. The speed distribution curve of the four dimensional mass $V_{M}$ is the effect of the mass $M$ on the total rotational speed of the galaxy. It is calculated by using the equation in which the gravitational force of the mass $M$ is equal to its component of the centrifugal force of rotation. The solution of this equation yields the speed distribution component $V_{M}$ of the four-dimensional mass $M$ and the value of the mass $M$. The publication [9] has presented the rotational speed distributions curves of the galaxy NGC 3198. The speed distribution curve of the galactic halo in the publication [9] corresponds to the speed distribution curve $V_{M}$ of four-dimensional mass $M$ in the publication [8]. In order to find out how well this four-dimensional model functions, the speed distribution curve $v_{M}$ was calculated by using two pairs of rotational radius and speed values. The conclusion was that the calculated distribution curve $V_{M}$ is a good match for the halo curve in the publication [9]. Furthermore, four rotational speed distribution curves $V_{M}$ were calculated using different values of the distance $X$, which yielded different values for the maximum radius of the galaxy. In this manner, the different galaxy models of the publication [2] were obtained, and therefore, this solution to the dark matter mystery has been proved. In the publication [10] it is calculated that $69 \%$ of the total mass energy of the Universe is dark energy that accelerates the Universe. This evaluation is due to the measurements of the wavelengths of light emitted from distant galaxies. The measurements have indicated that the wavelengths have become longer. The wave- 
length of light has shifted towards red. It is inferred that the redshift results from the speed of increasing distances of the far-away galaxies. The whole Universe is expanding at an increased rate of acceleration. In this study the model of the Universe is the surface volume of the four-dimensional spherical Universe. This type of structure of the Universe creates a similar redshift as measured. The cause of the redshift in this model of the Universe is its structure, and therefore, there is no accelerating expansion of the Universe. There is a Big Bang which is not very big, followed by deceleration, and at present, no expansion at all.

\section{The Universe in Four Distance Dimensions}

In the following, a simple method has been presented to see the space of four distance dimensions, and to perform calculations in it. In Figure 1, a box is drawn in the three-dimensional space $x, y, z$. On the left side of Figure 2, the same box is drawn in the two-dimensional space $z, x$ and on the right side it is drawn in the two-dimensional space $z, y$. There is still a third possible set of coordinates $x, y$, but it is not needed to determine the shape of the box. Here, it is seen that a structure of three dimensions can be drawn in the two-dimensional coordination system. In the same manner, a structure of four-dimensions can be drawn in coordination with the three dimensions $x, y, z, y, z, x^{\prime}, x, z, x^{\prime}, x, y, x^{\prime}$. If the structure is simple, it is possible that only one two-dimensional coordination system is needed to determine the form of the structure. Figure 3 presents the

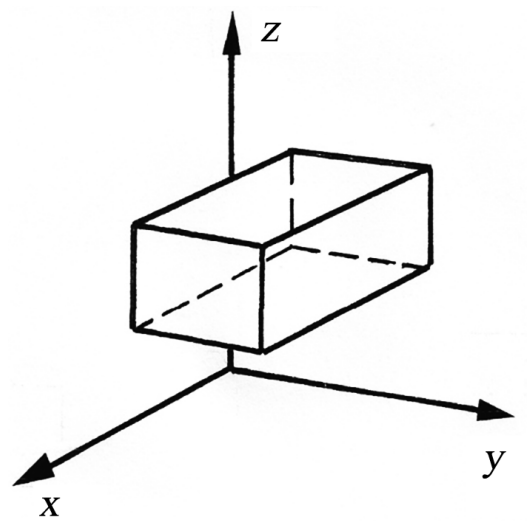

Figure 1. A box is drawn in the coordination system of three dimensional space $x, y, z$.
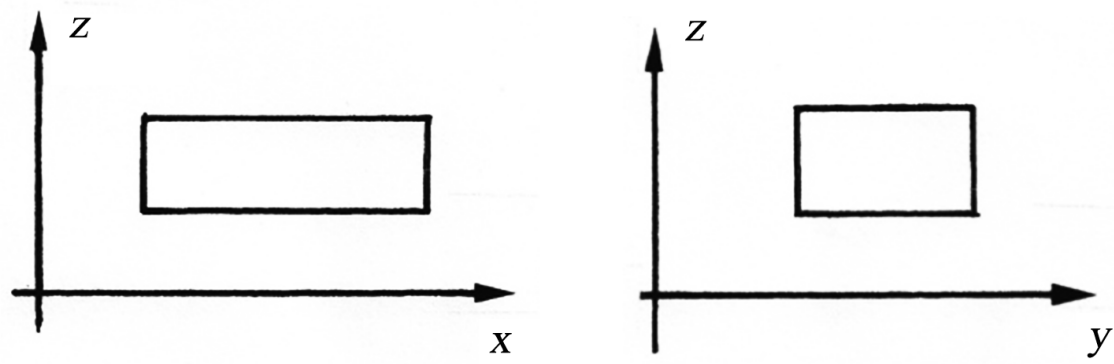

Figure 2. On the left side of the figure, the box is drawn in the coordination plane of two-dimensional space $x, z$ and on the right the box is drawn in the coordination plane $y, z$. 

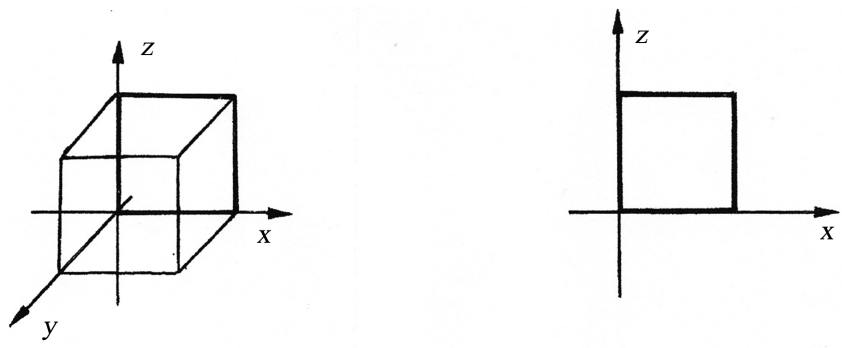

Figure 3. On the left side of the figure, there is a cube drawn in a coordination system of three-dimensional space $x, y, z$, and on the right is its cross-section on the $x, z$ plane. The thick-line square in the cube is the location of the cross-section. The cube on the left is located on the right in the square so that the volume of the cube is the area of the square on the right, and the four sides of the cube, which are at right angle to the cross-section square, are the four thick lines of the square on the right. This technique is used in the following mathematics of the four-dimensional space.

technique which is used in the mathematics of the four-dimensional space. Figure 4 and Figure 5 present the technique to see the four-dimensional structures in the three-dimensional coordination system.

In Figure 5, a sphere of four distance dimensions (right side) has been constructed from a sphere of ordinary three distant dimensions (left side). The ordinary dimensions are $x, y, z$ and the fourth dimension $x^{\prime}$. On the left side of Figure 5, there is a sphere of ordinary $x, y, z$ dimensions, and on the right side, there is a sphere of four dimensions $X, Y, Z, X^{\prime}$ which is presented as its $y, Z, X^{\prime}$ section. The cross-sections of both spheres on the $y, z$ plane are the same. The fourth-dimension $x^{\prime}$ is zero $\left(x^{\prime}=0\right)$ within the whole three-dimensional sphere on the left side. Therefore, the whole three-dimensional sphere (left side) is in the four-dimensional sphere (right side) on the $y, z$ cross-section plane, in which the fourth-dimension $x^{\prime}$ is zero $\left(x^{\prime}=0\right)$. The surface area of the three-dimensional sphere (left side) is at the distance $R$ from the center with all angle values of the coordination system (two angles and distance from center $R$ ). Thus, the surface area of the three-dimensional sphere (left side) is at the circumference line of the $y, z$ cross-section of the four-dimensional sphere (right side). The increased thickness of the circumference line indicates the location of the area of the three-dimensional sphere. Similarly, the volume of the three-dimensional sphere (left side) is located at the area of the $y, z$ cross-section of the four-dimensional sphere (right side). The four-dimensional sphere (right side) is formed by rotating the $y, z$ cross-section around the axis A-B half way as shown by the arrow. By doing so, the circumference line of the $y, z$ cross-section, which is the surface area of the three-dimensional sphere, creates a volume to the fourth power at the distance $R$ from the center of the four-dimensional sphere. The surface layer around the four-dimensional sphere is ordinary volume, and in the figure, the thick surface line of the sphere indicates volume. Within the $y, z$ cross-section, there is a volume of the four-dimensional sphere, and as the $y, z$ cross-section rotates around the A-B axis, different positions of the cross-section rotation indicate volumes of the four-dimensional sphere. Therefore, the third power 

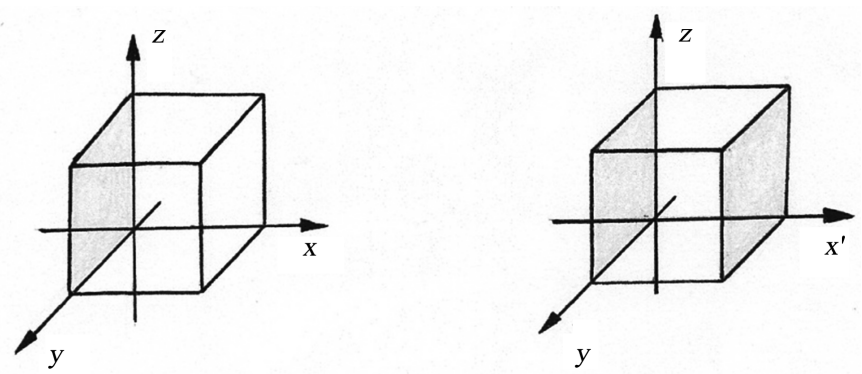

Figure 4. On the left of the figure, there is a three-dimensional $x, y, z$ section of four-dimensional $x, y, z, x^{\prime}$ cube, and on the right of the figure, there is a $y, z, x^{\prime}$ section of the same four-dimensional $x, y, z, x^{\prime}$ cube. Both cubes have the same side on the $y, z$ plane. Therefore, the volume between the two dark surface sides of the cube on the right is in the cube on the left in the area of the dark surface side on the $y, z$ plane. The three-dimensional coordination system of the cube on the right is $y, z, x^{\prime}$ and the whole $x$ axis is in the zero point of the coordinate axes. All six sides of the cube on the left can be determined to have the same volume, and the total volume of the six sides of the cube is six times the volume of the cube. In the same manner, the $x, y, z$ section of the four-dimensional $x, y, z, x^{\prime}$ sphere has a surface area which is three-dimensional volume. In this study, this type of surface area is a model of the Universe, and it produces a good fitting with the redshift measurements, proving it to be true.
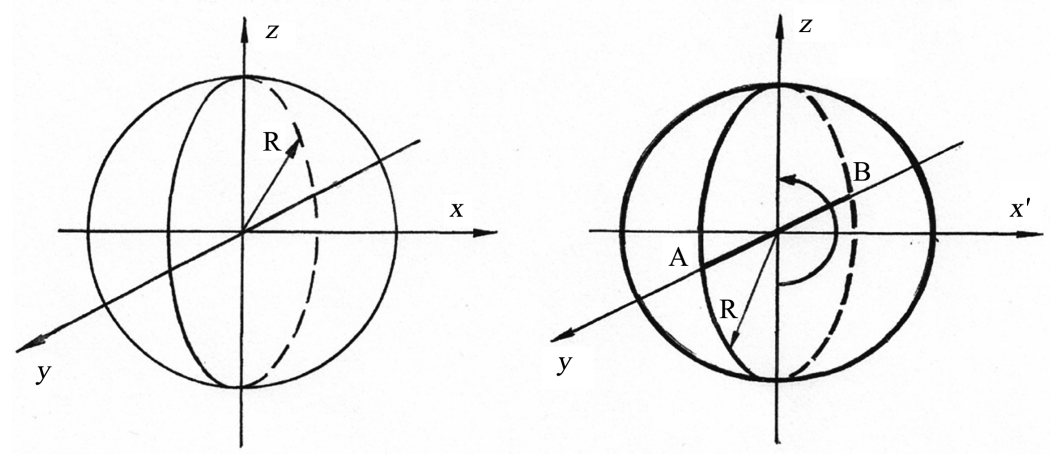

Figure 5. On the left side of the figure, there is an ordinary three dimensional sphere and, on the right side a four-dimensional sphere. The coordinate axes on the left side are $x, y, z$ and $y, z, x^{\prime}$ on the right side, and the whole $x$ coordinate axis is located on the right side in the zero point of the coordinate axes. The $y, z$ cross-sections of both spheres are the same. The circumference line of the $y, z$ cross-section of the four-dimensional sphere on the right side corresponds to the surface area of the three-dimensional sphere on the left side, and the area of the $y, z$ cross-section of the four-dimensional sphere on the right side corresponds to the volume of the three-dimensional sphere on the left side. The four-dimensional sphere is formed by rotating the $y, z$ cross-section around the axis A-B half a rotation (half circle arrow), in the same manner as forming the ordinary three-dimensional sphere.

volume of the four-dimensional sphere is infinite, and the finite volume is to the fourth power. The volume units are $\mathrm{m}^{3}$ and $\mathrm{m}^{4}$. This is the theory of the fourdimensional space. However, it is most interesting to apply this theory to the construction of the Universe. The redshift of distant galaxies can be explained with this theory. The surface area of the four-dimensional sphere is a finite volume, which, in theory, can be the Universe.

By studying the $Z, X^{\prime}$ cross-section of four-dimensional sphere (right side), it 
can be seen that at the intersection point of $z$ axis and the sphere the direction of the surface is $x^{\prime}$ axis, and at the intersection point of $x^{\prime}$ axis and the sphere the direction of the surface is $z$ axis. In the model of the Universe this means that the light that travels between these two points disappears because of the turning of the dimension axes. In the surface volume of the four-dimensional sphere, the turning of the dimension axes acts in the same manner as in this two-dimensional cross-section. This is the theory, and the redshift of distant galaxies may be the evidence for this theory. The accelerating redshift rate at the farthest galaxies fits to this model of the Universe. Another factor that fits to this theory of the Universe is the Big Bang as the beginning of the Universe. It is known that the Universe is the same in every direction. If the Universe is a three-dimensional ball, the point of the Big Bang could be seen in the Universe, and the Universe would be different in different directions. It can be thought that the beginning of the four-dimensional spherical Universe is an explosion of the four-dimensional mass concentration. The shape of the Universe as a surface volume of the fourdimensional sphere could be the shock wave of the Big Bang explosion. The beginning point of the Universe, the Big Bang, cannot be seen in the Universe of the four-dimensional sphere because in the surface volume of the four dimensional sphere all the three dimensions are at right angle to the direction of the point of the Big Bang. Therefore the Universe is the same in every direction.

Calculation of the volume of the Universe:

1) Figure 4 presents the principle of the four-dimensional spherical Universe. In the same manner as in Figure 4, the section of the four-dimensional $x, y, z, x^{\prime}$ cube has six surface side volumes, the four-dimensional sphere has similar surface volume, which in this study is a model of the Universe.

2) The $x, z$ cross-sections are the same in Figure 5 left and Figure 6.

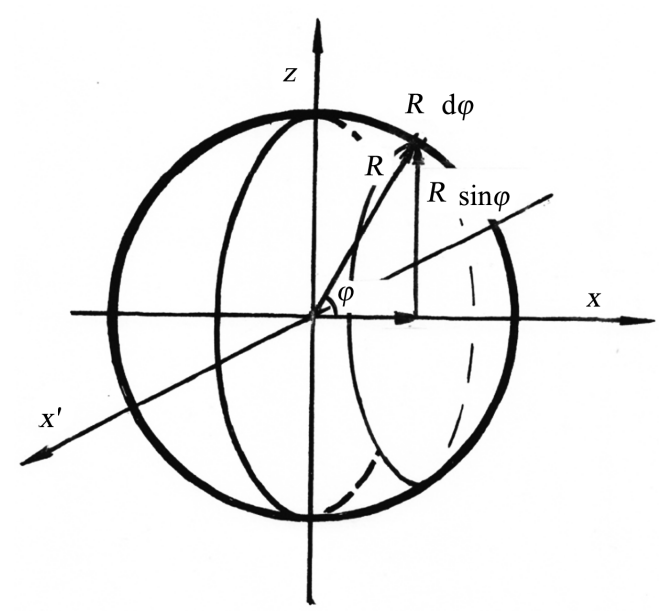

Figure 6. The figure presents a three-dimensional $x, Z, X^{\prime}$ section of the four-dimensional $x, y, z, x^{\prime}$ sphere. The surface layer of the four-dimensional sphere is the model of the Universe. The thick circle line at the radius $R$ is the area of the sphere in Figure 5 left. The volume of the Universe is calculated by rotating the minimal area $R \mathrm{~d} \varphi$ ( $\varphi$ radians) half a rotation along the solid circle line. 
3) The whole surface area of the three-dimensional sphere in Figure 5 left is located at the circumference of the $x, z$ cross-section of the four-dimensional sphere in Figure 6. This is using the coordination system of two angles and the distance from the center $R$, and the technique of Figure 3. The thick circumference line indicated area.

4) The area within the length of the circumference $R \mathrm{~d} \varphi$ in Figure 6 is

$$
\mathrm{d} A=2 \pi R \sin \varphi \cdot R \mathrm{~d} \varphi=2 \pi R^{2} \sin \varphi \mathrm{d} \varphi
$$

5) Half a rotation (solid line) of the area is the increment of the volume

$$
\mathrm{d} V=2 \pi R^{2} \sin \varphi \mathrm{d} \varphi \cdot \pi R \sin \varphi=2 \pi^{2} R^{3} \sin ^{2} \varphi \mathrm{d} \varphi
$$

6) Volume of the Universe

$$
2 V=4 \pi^{2} R^{3} \int_{0}^{\pi / 2} \sin ^{2} \varphi \mathrm{d} \varphi
$$

Figure 7 presents four-dimensional $x, y, Z, x^{\prime}$ sphere as its two-dimensional cross-section $x, z$. The model of the Universe is the cover volume of the fourthdimensional sphere at the distance $R$ from the center. The photon of light has been emitted at point $\mathrm{A}$, and it has been observed at point $\mathrm{B}$. The angle between the points $\mathrm{A}$ and $\mathrm{B}$ is $\varphi$ radians. At point $\mathrm{A}$ the photon's energy is $E$, and as the photon travels to point $\mathrm{B}$, its energy is $E \cos \varphi$. This is because all the three surface volume dimensions must be at right angle to the radius vector $R$ of the four-dimensional sphere. Therefore, it can be concluded that the photon, which has been emitted at point $\mathrm{A}$, as it travels to point $\mathrm{C}$, will completely disappear. This is a theory, but the next part of this chapter will demonstrate that the theory functions correct within the calculations of the redshift of light in the Universe.

The increase of wavelength in the Universe was calculated according to the decrease of the energy of the light photon. Basic equations of light are speed of light and energy of the light photon

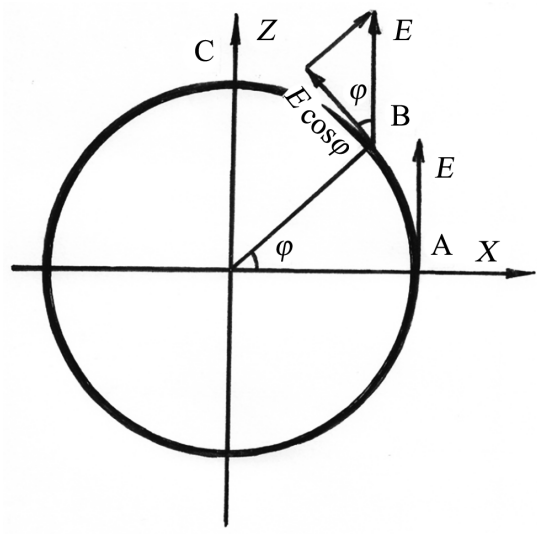

Figure 7. This figure features two-dimensional $x, z$ cross-section of the four-dimensional $x, y, z, x^{\prime}$ sphere. The model of the Universe is the three-dimensional volume cover of the four-dimensional sphere. The three-dimensional volume is presented as the circumference of the radius $R$. As the light photon travels from point A to point $\mathrm{B}$, its energy decreases from $E$ to $E \cos \varphi$. 


$$
\begin{aligned}
& c=\lambda_{0} \times f \\
& \lambda_{0}=h c / E
\end{aligned}
$$

The equation of the wavelength of light in Figure 7

$$
\lambda=\frac{h c}{E \cos \varphi}
$$

in which

$h$ : Planck's constant;

c. Speed of light;

$E$ : Photons energy at point A in Figure 7;

$E \cos \varphi$ Photons energy at point B;

$\lambda_{0}$ : Ordinary wavelength;

$\lambda$ : Redshift wavelength.

The wavelength of the above equation was fitted to the model of the Universe which was constructed according to the measurements of redshift in the Universe. The redshift measurements were published in scientific journals and as well as in newspapers around the world, and here was used the model of the Universe in publication [10]. The above equation of the wavelength of light fitted this model of the Universe, but was not good enough, and therefore a component of the expansion of the Universe was added to the equation, and then it did match.

The equation of the approximate increase of wavelength in the Universe is

$$
\lambda=\frac{h c}{E \cos \varphi}+0.0033 \frac{h c}{E} \varphi
$$

The fitting of the wavelength model (Figure 8) to the model of the Universe in publication [10] (Figure 9) is as follows:

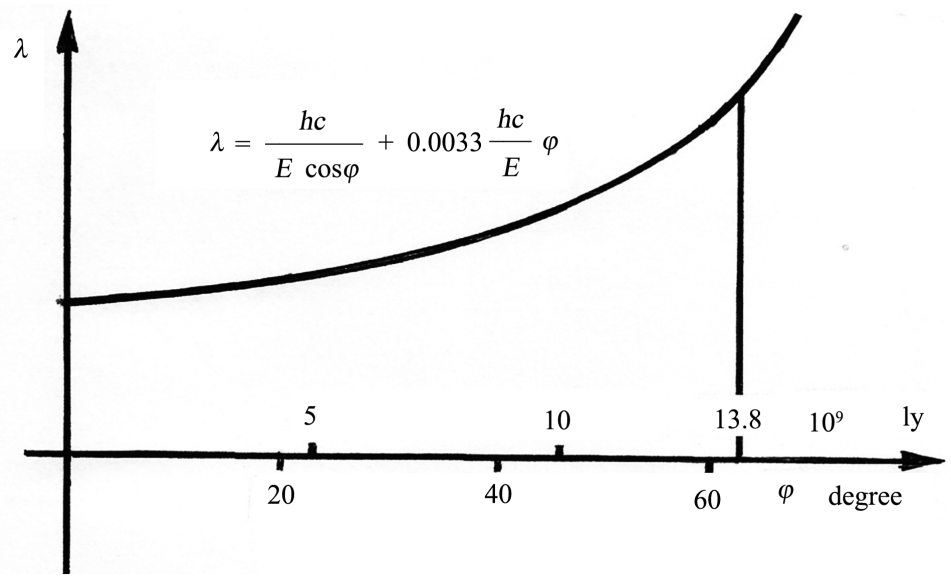

Figure 8. The vertical axis $\lambda$ is the wavelength of the light photon emitted at point $\mathrm{A}$ in Figure 7, beneath the horizontal axis is the angle $\varphi$ of the Universe in Figure 7, and above the horizontal axis is the distance in light years. The redshift in the Universe is the increase of wavelength according to the equation $\lambda-\lambda_{0}$. The redshift has two components: the first component is the effect of $E \cos \varphi$ in Figure 7, and the second component is the effect of the approximate expansion speed of the Universe. 


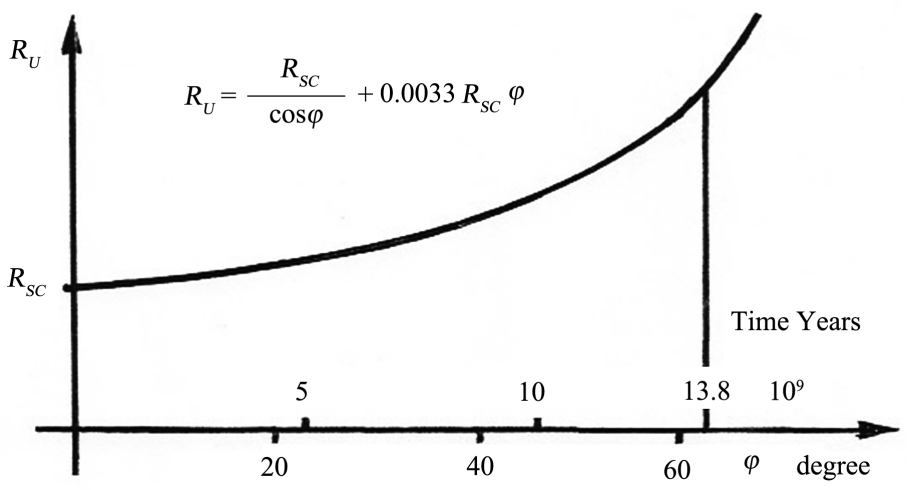

Figure 9. The model of the Universe in the publication [10], in which the radius of the Universe is $R_{U}$ and the scale factor $R_{s c}$ determines the scale of the $R_{U}$ radius axis.

The time derivative of the radius of the Universe $R_{U}$ is the expansion velocity of the Universe, and it is directly proportional to the change of wavelength. Angle $\varphi$ is constant multiplied by time $C \times t$ and the equation well-suited for to the model of the Universe is

$$
R_{U}=\frac{R_{S C}}{\cos C t}+0.0033 R_{S C} C t
$$

derivative in the textbook

$$
\begin{aligned}
& \frac{\mathrm{d}\left(\cos ^{-1} C t\right)}{\mathrm{d}(\cos C t)}=-\cos ^{-2} C t, \quad \frac{\mathrm{d}(\cos C t)}{\mathrm{d} C t}=-\sin C t, \quad \frac{\mathrm{d}(C t)}{\mathrm{d} t}=C \\
& \Rightarrow \frac{1}{\cos C t}=\frac{C \sin C t}{\cos ^{2} C t}
\end{aligned}
$$

derivative of the radius of the Universe is

$$
\frac{\mathrm{d} R_{U}}{\mathrm{~d} t}=\frac{R_{S C} \sin C t}{\cos ^{2} C t} \cdot C+0.0033 R_{S C} \cdot C
$$

This equation fits well to the equation of wavelength Equation (5) using the fittings

$$
\begin{gathered}
C t=\varphi \\
R_{S C} \cdot C=1 \Rightarrow \frac{h c}{E}=2.9 \\
\lambda-\frac{h c}{E}=\frac{\mathrm{d} R_{U}}{\mathrm{~d} t}=\frac{R_{S C} \sin \varphi}{\cos ^{2} \varphi} \cdot C+0.0033 R_{S C} \cdot C
\end{gathered}
$$

Both equations, Equation (5) and Equation (7) are very similar between angels 0 - 60 degrees, and therefore, the time derivative of the model of the Universe Equation (7), which is the expansion speed of the Universe, is almost directly proportional to the equation of the change of wavelength Equation (10). The change of wavelength is the redshift.

The wavelength of the redshift in the Universe is presented in Figure 8 with a model of the approximate expansion speed of the Universe. Matching of the above wavelength equation means that the theory of the Universe appears to be 
correct. The range of the wavelength measurements is so large that the fitting cannot be coincidental. A more accurate analysis of the expansion speed of the Universe is in Figure 10. The expansion speed of the Universe in publication [10] by The Swedish Academy of Science is presented so that our time is $13.8 \times$ $10^{9}$ years after the Big Bang at which point in time, the speed is at a high rate of acceleration. This is because the mass of the whole Universe is in the point of Big Bang, and therefore it cannot move, while our galaxy moves. The Universe of the four-dimensional sphere is different. Our galaxy and the galaxies of the Big Bang are both at the surface area of the sphere of the Universe, and they are both moving in opposite directions. Therefore, the galaxies of the Big Bang move and our galaxy is near stationary. In Figure 10 the zero point is our time. The equation above is Equation (10), in which the term $0.0033 R_{s c}$ has been neglected because it is too small to have any effect. The equation below is the effect of the turning of the Universe dimensions in Figure 7, Equation (4).

Calculation of the volume of the Universe continues, Equation (3)

$$
2 V=4 \pi^{2} R^{3} \int_{0}^{\pi / 2} \sin ^{2} \varphi \mathrm{d} \varphi
$$

Approximate calculation of the integral

$$
2 V=4 \pi^{2} R^{3} \sum_{0}^{\pi / 2} \sin ^{2} \varphi \cdot \Delta \varphi
$$

in which the increment of the angle is $\Delta \varphi=\pi / 20$, and the first angle of calculation $\pi / 40$. The radius of the four-dimensional sphere $R$ in Figure 7 can be calculated based on Figure 8. The maximum distance of measurement is $13.8 \times 10^{9} \mathrm{ly}$ which corresponds to the angle $\varphi=62.7^{\circ}$, and the angle $\varphi=360^{\circ}$ corresponds to the distance $79.2 \times 10^{9}$ ly which is the circumference of the four-dimensional

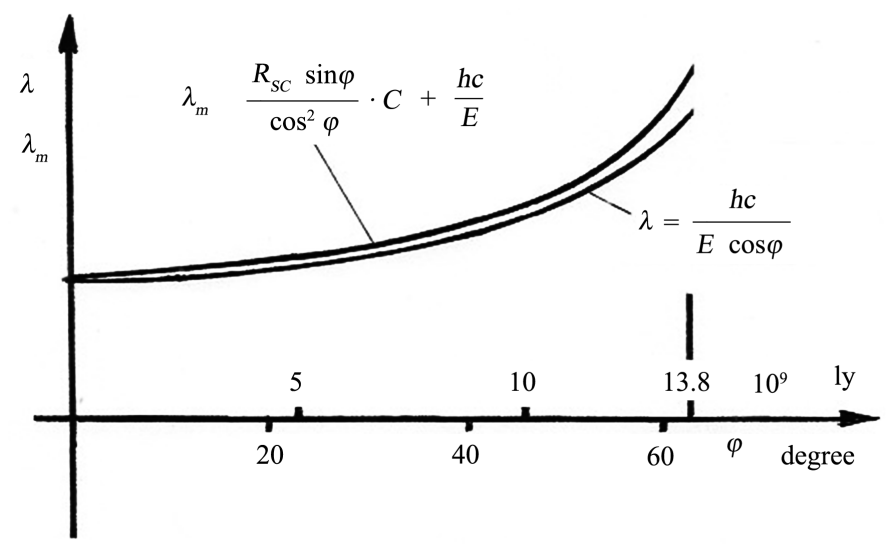

Figure 10. Measured wavelength is $\lambda_{m}$ Equation (10) and the increase of wavelength according to Equation (4) is $\lambda$, which is the effect of the turning of the University dimensions in Figure 7. The difference of these wavelengths is directly proportional to the expansion speed of the Universe. The difference of the wavelengths $\lambda_{m}-\lambda$ is increasing at the point of the longest measuring range $13.8 \times 10^{9}$ ly which indicates the Big Bang. The expansion speed at that point is not very high and it seems that the Big Bang is not very big. Before that point the expansion speed of the Universe decelerates. 
sphere, and its radius is $R=79.2 \times 10^{9} \mathrm{ly} / 2 \pi=12.6 \times 10^{9} \mathrm{ly}$. The summation of the square of sine is

$$
\sum_{0}^{\pi / 2} \sin ^{2} \varphi=5
$$

and the volume of the Universe is

$$
2 V=4 \pi^{2} R^{3} \sum_{0}^{\pi / 2} \sin ^{2} \varphi \cdot \Delta \varphi=4 \pi^{2} \times 12.6^{3} \times 5 \times \pi / 20 \times 10^{27} \mathrm{ly}^{3}=62 \times 10^{30} \mathrm{ly}^{3}
$$

The volume of the center sphere of the Universe is

$$
\frac{4}{3} \pi R^{3}=\frac{4}{3} \pi \times 12.6^{3} \times 10^{27} 1 \mathrm{y}^{3}=8.38 \times 10^{30} 1 \mathrm{y}^{3}
$$

The volume of the Universe divided by the volume of the center sphere of the Universe is $62 / 8.38=7.4$.

This result can be compared with the corresponding value of the four-dimensional cube. If the center cube of the $x, y, z$ space in Figure 4 had a volume of 1 $\mathrm{m}^{3}$, then six sides of the center cube would have a volume of $6 \mathrm{~m}^{3}$. These pairs of volume sides are the spaces $y, z, x^{\prime}, x, z, x^{\prime}$ and $x, y, x^{\prime}$. Because this is a fourdimensional cube, there must also be a pair of volume sides $x, y, z$ which corresponds to the center cube $x, y, x^{\prime}$. Then the volume of the sides around the four-dimensional cube is thus $8 \mathrm{~m}^{3}$. The volume of the sides around the fourdimensional cube divided by the volume of the center cube is $8 / 1=8$. The ratio for the Universe 7.4 seems to be correct.

\section{Conclusions}

This study is a research of the Universe in four dimensions. This is a continuation of the previous research "Galaxy rotation in four distance dimensions", publication [8]. In that research it was proven that great masses in the four-dimension generate the rotation of galaxies. The prevailing theory has been that the galaxy rotation would be a result of halo mass round about the galaxy. The issue with this theory is that the halo mass must be very inactive before unknown dark matter, which despite massive search has not been found. In publication [8] the hypothetical dark matter is four-dimensional. Because of four dimensions this dark matter decreases to the power of three, as the ordinary three-dimensional matter decreases to the power of two. Due to the substantial decrease of gravitational force in large distances, the mass of the four-dimensional dark matter must be much greater than the ordinary mass to be able to generate the galaxy rotation. An ordinary distance within galaxy rotation could be for instance $10^{20} \mathrm{~m}$ and the decrease in gravitational force of ordinary dark matter is then in the order of $\left(10^{20}\right)^{2} \mathrm{~m}^{2}=10^{40} \mathrm{~m}^{2}$, but the decrease of the four-dimensional mark matter is $\left(10^{20}\right)^{3} \mathrm{~m}^{3}=10^{60} \mathrm{~m}^{3}$. Therefore, there must be $10^{20}$ times the amount of the four-dimensional dark matter compared to the ordinary matter in order to generate the same galaxy rotation speed. The Universe in four dimensions must be considerably more massive than our Universe of three dimensions. It is probable 
that the mass of the four-dimensional Universe is concentrated into a spherical shape formation, and our three-dimensional Universe is the surface layer volume of that sphere. The gravitational force of the great mass of the four-dimensional Universe has no effect on our three-dimensional Universe because its force of gravity is at the right angle to the three dimensions of our Universe, except if the four-dimensional mass concentration is very near. Furthermore, because the force of gravity of four-dimensional matter decreases at a high rate, it disappears at large distances.

In Figure 11, the progress of research and testing the hypothesis is presented in the same manner as it is in Karl Poppers's book [11]. The first step, the hypothesis, is that the redshift measurements from distant galaxies are due to the shape of the four-dimensional Universe, and also the expansion of the Universe. The second step is derivation of the equations. This phase of proceeding involves the derivation of mathematics of the four-dimensional space. In the three-dimensional section of the four-dimensional space, area indicates volume and line indicated area. Thereafter, the equation of the light photon travelling in the spherical shape surface volume of the four-dimensional Universe can be derived. The third step is the test prediction which is that the equation of the light photon travelling in the surface volume of the four-dimensional Universe (Figure 7) is the same as the measured redshift values in the Universe. The fourth step is the measurements, which can be calculated based on the model of the Universe in publication [10] (Figure 9). A Perfect fitting for that model was Equation (6), and the derivative of that equation (Equation (7)) should be the equation of the light photon travelling the surface volume of the four-dimensional spherical Universe (Equation (5), Figure 8). The fifth step is the analysis of the result. In the analysis, the derivative of the measured theoretical radius of the Universe (Figure 9, Equation (6)) is compared to the wavelength of the light photon travelling in the Universe. The first fitting is performed with an approximate model of expansion of the Universe (Figure 8, Equation (5)), and the second more accurate fitting is performed with a model of the Big Bang (not very big) a decelerating

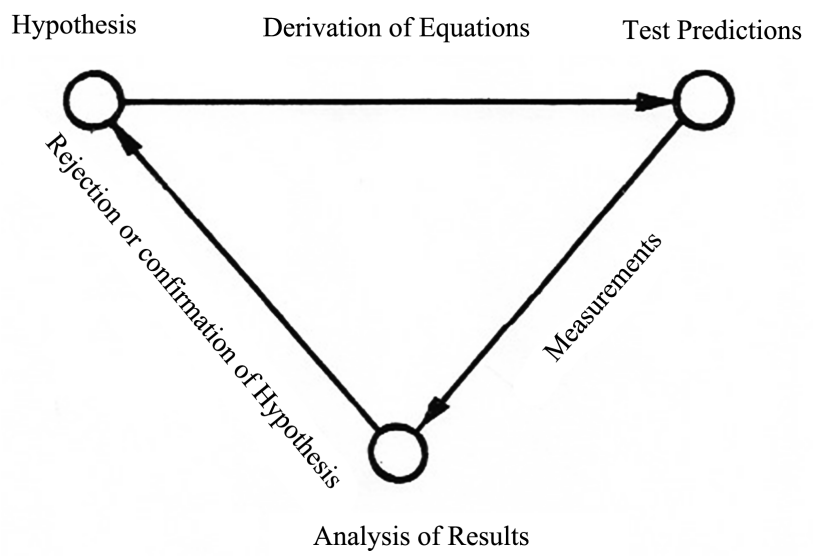

Figure 11. The progress of testing the hypotheses of the Universe of four dimensional sphere publication Karl Popper [11]. 
expansion (Figure 10). In these models of the Universe, dark energy which is $69 \%$ of the total mass energy of the Universe does not exist.

The final step is rejection or confirmation of the hypotheses, and it was found that the equation of the derivative of the radius of the theoretical Universe, Equation (7), fits well to the equation of wavelength, the first fitting Equation (5) and the second fitting Equation (4), Figure 10 using the fittings of Equation (8) Equation (10). The first round of the progress triangle (following the arrows) is "Galaxy Rotation in the Space of Four Dimensions" [8], and this study is the second round. This method of progress was used for the solution to the Hill's equation. The famous British Nobel laureate A. V. Hill invented this equation in 1938. Within four rounds that problem was solved, publications [12] [13] [14] [15].

The theoretical model of the four-dimensional Universe has been proved as follows: the turn of the surface volume dimensions of the theoretical four-dimensional spherical Universe results in the major redshift Equation (4), to which the slight redshift effect of the expansion of the Universe is added. The redshift summation Equation (5) was approximately equal to the measured redshift Equation (10) which was calculated based on the model of the Universe in publication [10]. Precise fitting is in Figure 10.

In publication [16] was presented an inflating balloon as a good analogy for understanding the expansion of the Universe. Because the Universe does not appear to have an edge or a center, and the inflating balloon also has no edge or center, it fits well with the model of the Universe. However, this model of the Universe has one major problem: the surface area of the balloon has two dimensions, but the Universe has three dimensions. The four-dimensional spherical Universe in this study has a surface volume at a certain radius from the center, and this surface volume has three dimensions. Therefore, the four-dimensional sphere is the only one that fits the model of the Universe. The problem with the age of the Universe $13.8 \times 10^{9}$ years is that it is too short. The age of the Universe is $365 \times 60 \times 60 \times 24 \times 13.8 \times 10^{9}=43 \times 10^{16}$ seconds and the time a star (speed $150 \mathrm{~km} / \mathrm{s})$ rotates around the center of galaxy $\left(R=5 \times 10^{20} \mathrm{~m}\right)$ may be $2 \times \pi \times 5$ $\times 10^{20} / 1.5 \times 10^{5}=2.09 \times 10^{16}$ seconds, and within 21.5 rotations the complex structure of the galaxy cannot be created by evolution. This problem is not in the model of the four-dimensional spherical Universe. The time $13.8 \times 10^{9}$ years in that model of the Universe is the time that light travels $63^{\circ}$ of the rotation around the center of the Universe. Figure 8 demonstrates that after the distance $13.8 \times 10^{9}$ light years the redshift curve increases very steeply, and therefore redshift measurements after that point are very difficult. In the model of the four-dimensional spherical Universe, also the Big Bang appears to be real, but it is not very big. The accurate fitting of Equation (10) yields the expansion of the Universe which first accelerates and then decelerates. The model of the Universe by NASA, publication [17], is available online. In that version, the radius of the Universe after the Big Bang is much larger than in the version by The Swedish Academy of Science, publication [10]. The redshift measurements do not indi- 
cate the total radius of the Universe, only the increase of the wavelength of light. The redshift in the Universe is the increase of wavelength according to the equation $\lambda-\lambda_{0}$. In the models of the Universe, publications [10] and [17], the redshift measurements indicate the increase of radius of the Universe. Equation (9) is in NASA model in the form

$$
R_{S C} \cdot C=0.203 \Rightarrow \frac{h c}{E}=0.203 \times 2.9=0.59
$$

in which the values are in the same ratio than as Equation (9). Otherwise these two models of the Universe are very similar.

\section{Conflicts of Interest}

The author declares no conflicts of interest regarding the publication of this paper.

\section{References}

[1] Ostriker, J.P., Peebles, P.J.E. and Yahil, A. (1974) The Size and Mass of Galaxies, and the Mass of the Universe. The Astrophysical Journal, 193, L1-L4. https://doi.org/10.1086/181617

[2] Rubin, V.C. (1983) The Rotation of Spiral Galaxies. Science, 220, 1339-1344. https://doi.org/10.1126/science.220.4604.1339

[3] Burrows, A. and Liebert, J. (1995) Probing Dark Matter. Nature, 373, 191-192. https://doi.org/10.1038/373191a0

[4] Moster, B.P., Somerville, R.S., Maulbetsch, C., van den Bosch, F.C., Maccio, A.V., Naab, T. and Oser, L. (2010) Constraints on the Relationship between Stellar Mass and Halo Mass at Low and High Redshifts. The Astrophysical Journal, 710, Article No. 2. https://doi.org/10.1088/0004-637X/710/2/903

[5] Behroozi, P.S., Wechsler, R.H. and Wu, H. (2012) The Rockstar Phase-Space Temporal Halo Finder and the Velocity Offsets of Cluster Cores. The Astrophysical Journal, 762, Article No. 2. https://doi.org/10.1088/0004-637X/762/2/109

[6] Panek, R. (2020) A Cosmic Crisis. Scientific American, 322, 30.

[7] Roos, M. (2003) Introduction to Cosmology Third Edition. John Wiley \& Sons, Inc., Hoboken.

[8] Rahikainen, A. (2020) Galaxy Rotation in the Space of Four Distance Dimensions. World Journal of Mechanics, 10, 83-92. https://doi.org/10.4236/wjm.2020.107007

[9] van Albada, T.S., Bahcall, J.N., Begeman, K. and Sanscisi, R. (1985) Distribution of Dark Matter in the Spiral Galaxy NGC 3198. The Astrophysical Journal, 295, 305-313. https://doi.org/10.1086/163375

[10] Jarnestad, J. (2019) Grapgics, the Royal Swedish Academy of Sciences, ML/HS. Helsingin Sanomat. https://www.hs.fi/tiede/art-2000006265660.html

[11] Popper, K. (2002) Conjectures and Refutations: The Growth of Scientific Knowledge. Routledge , Milton Park.

[12] Rahikainen, A. (2019) Constant Power Solution of Hill's Equation. Book Publisher International, Hooghly. https://doi.org/10.9734/bpi/mono/978-81-940613-2-8

[13] Rahikainen, A. and Virmavirta, M. (2019) Hill's Equation in Arm Push of Shot Put and in Braking of Arm Rotation. Advances in Applied Science and Technology, 6, Chapter 5 . 
[14] Rahikainen, A. and Virmavirta, M. (2014) Constant Power Model in Arm Rotation-A New Approach to Hill's Equation. World Journal of Mechanics, 4, 157-169. https://doi.org/10.4236/wjm.2014.46018

[15] Rahikainen, A. and Virmavirta, M. (2012) Modeling the Force-Velocity Relationship in Arm Movement. World Journal of Mechanics, 2, 90-97. https://doi.org/10.4236/wjm.2012.22011

[16] Lineweaver, C.H. and Davis, T.M. (2007) Misconceptions about the Big Bang. Scientific American, 292, 36-45.

[17] Wollack, E.J. (2012) Timeline of the Universe Image, NASA/WMAP Science Team. https://map.gsfc.nasa.gov/media/060915/index.html

\section{List of Variables}

Dimensions of ordinary space

Fourth distance dimension

$X^{\prime}$

Four-dimensional mass

$M$

Distance of the four-dimensional mass from center of galaxy

$X^{*}$

Speed distribution component of the four-dimensional mass

$V_{M}$

Radius of the four-dimensional spherical Universe

Coordination angle of the four-dimensional spherical Universe

$R$

Distance, Light years

$\varphi$

Time, Years

ly

Energy of light photon

$t$

Planck's constant

E

Speed of light

$h$

Volume of the four-dimensional spherical Universe

Radius of the three-dimensional Universe

V

Scale factor

$R_{U}$

Ordinary wavelength

$R_{s c}$

Redshift wavelength

$\lambda_{0}$

Measured wavelength 